\title{
Coupling to photonic crystal fibers
}

Hougaard, Kristian G.; Bjarklev, Anders Overgaard; Knudsen, Erik; Libori, S.E. Barkou; Riishede, Jesper; Skovgaard, Peter M. W.; Broeng, Jes

Published in:

Optical Fiber Communication Conference and Exhibit, 2002. OFC 2002

Link to article, DOI:

10.1109/OFC.2002.1036607

Publication date:

2002

Document Version

Publisher's PDF, also known as Version of record

Link back to DTU Orbit

Citation $(A P A)$ :

Hougaard, K. G., Bjarklev, A. O., Knudsen, E., Libori, S. E. B., Riishede, J., Skovgaard, P. M. W., \& Broeng, J. (2002). Coupling to photonic crystal fibers. In Optical Fiber Communication Conference and Exhibit, 2002. OFC 2002 (pp. 627-628). IEEE. https://doi.org/10.1109/OFC.2002.1036607

\section{General rights}

Copyright and moral rights for the publications made accessible in the public portal are retained by the authors and/or other copyright owners and it is a condition of accessing publications that users recognise and abide by the legal requirements associated with these rights.

- Users may download and print one copy of any publication from the public portal for the purpose of private study or research.

- You may not further distribute the material or use it for any profit-making activity or commercial gain

- You may freely distribute the URL identifying the publication in the public portal 
Coupling to Photonic Crystal Fibers

Kristian G. Hougaard, Anders Bjarklev, Erik Knudsen, Stig Barkou Libori, and Jesper Riishede, Research Center COM, Technical University of Denmark, Building 345V, Kgs. Lyngby DK-2800, Denmark, Email: kgh@com.dtu.dk

Peter M.W. Skovgaard, Jes Broeng, Crystal Fibre A/S, Blokken, 84, DK-3460 Birkerød, Email. jb@crystal-fiber.com

\section{Introductlon}

Photonic crystal fibers (PCFs) form a novel class of optical fibers that have attracted a considerable amount of interest due to their unconventiona properties. ${ }^{1-3}$ The main difference between standard fibers and PCFs is that the cladding in PCFs may consist of pure silica with an array of air holes running along the length of the fiber. PCFs are divided into two groups, depending on the guiding mechanism. The light is guided either by modified total internal reflection (M-TIR) or by the photonic bandgap (PBG) effect. ${ }^{1}$ In this presentation, we will focus on index-guiding PCFs. This choice is due to the larger availability of index-guiding PCFs and relatively close resemblance to standard fibers. For almost any application, if PCFs are to be a competitive alternative or complement to standard optical fibers, an in-depth understanding of these fiber's coupling properties are necessary. However, at present, very few studies of coupling issues of PCFs have been presented in literature. ${ }^{2,}$ In this work, we present a general treatment of coupling to PCFs, focusing on fundamental aspects of the PCF mode distributions, such as their overlap to gaussian beams and the angular de pendency of transversal offset.

\section{PCF mode fundamentals}

The PCFs considered in this article all have triangular cladding structures as seen in Fig. 2. This is today the most commonly used structure for PCFs and it arises from a close packing of circular silica tubes. The core is made by replacing one tube with a solid silica rod--see Fig. 1.

Generally, coupling losses have three fundamental sources: Mode mismatch, Fresnel reflections on the interface between the fibers, and misalignment of the fibers. We will here mainly focus on mode mismatch and as a first approximation Fresnel reflections are assumed negligible. This enables us to treat the coupling between the fibers as an overlap integral between two mode profiles. This approximation is justifiable because Fresne reflections are caused by changes in the refractive
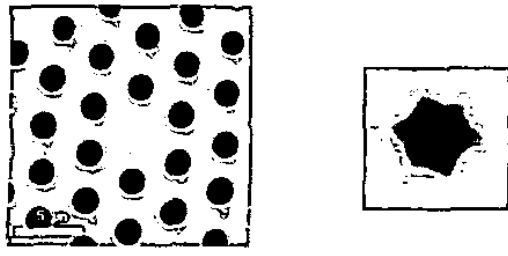

ThGG11 Fig. 1. Left: Scanning electron micrograph of PCF cross-section. Right: Fundamental mode field distribution having an apparent hexagonal shape. index, and for the considered PCF to SIF coupling, the majority of the light is confined to the core, where we find a glass-glass interface. Some of the problems related to PCF coupling are illustrated in Fig. 2, where three examples of fundamental mode profiles are shown above the fibers. While the mode profiles of standard fibers, such as step index fibers (SIF), are circular symmetric, the mode profiles of PCFs inherit the hexagonal symmetry of the index distribution of the PCF. This difference in the mode shape causes a loss of energy, when coupling between SIFs and PCFs. The two PCF modes illustrate the large variation in mode profiles possible for PCFs. The mode profiles have a strong dependence on the hole size, the pitch, and the frequency. The rightmost profile in Fig. 2 is an example of the light being strongly confined to the core. This is caused by very large holes in the PCF (the specific fiber has $\mathrm{d} / \Lambda=0.9$ ), and the mode profile becomes almost circular symmetric. The mode in the middle is calculated for a PCF with a normalized hole size, $d / \Lambda=0.3$, and a relatively low frequency allowing the light to spread out between the holes, into the cladding. Because of this large spectral mode variation, it is necessary to describe, in detail, the properties of the mode profiles.

The coupling coefficients between two fibers are calculated as overlap integrals of the mode profiles of the fibers. The exact overlap between two electromagnetic fields is given by (1), where $\mathrm{E}$ and $\mathrm{H}$ corresponds to the electric and the magnetic fields, respectively, and subscripts in and out corresponds to the electromagnetic fields in the two coupled fibers, respectively. Integration is performed across the full cross section, $\mathrm{A}$, of the fibers. As a simplification, the overlap integrals in this article may be calculated as (2)

$$
\begin{gathered}
c=\frac{\int_{A}\left(E_{\text {in }} \times H_{\text {out }}+E_{\text {out }} \times H_{\text {in }}\right) \cdot d a}{\int_{A}\left(E_{\text {in }} \times H_{\text {in }}+E_{\text {out }} \times H_{\text {out }}\right) \cdot d a} \\
c=\frac{\int_{A}\left(H_{\mathrm{pcf}} \cdot E_{\text {sif }}\right) \cdot d a}{\sqrt{\int_{A_{1}} H_{\mathrm{pcf}_{1}^{2}}^{2} \mathrm{da}_{1} \cdot \int_{A_{2}} E_{\text {sif }}^{2} \mathrm{da}_{2}}} .
\end{gathered}
$$

Equation (2) gives an exact value, when the two fields have the same intrinsic impedance, which is the case, if the two fields propagate with the same propagation constant, i.e., if they have the same modal index.

3. Coupling from PCFs to gaussian beams and step-index fibers

The first step in the analysis is to compare the PCF modes with gaussian shaped modes. This

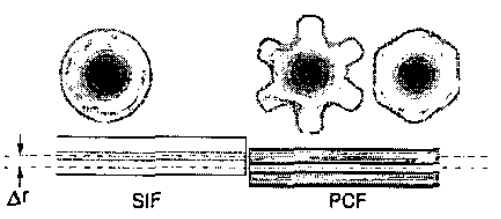

ThGG11 Fig. 2. Schematic drawing of a fiber splice between a step-index fiber (SIF) and a PCF. The transversal offset is denoted $\Delta r$. Examples of the considered mode profiles are shown above the fibers. The PCF modes are calculated at different wavelengths and the figure illustrates the large variation in PCF mode shape and the fundamental difference between the mode profiles of PCFs and SIFs. provides a point of reference, when comparing PCFs to standard fibers. We compare a PCF mode to a gaussian mode by calculating the specific gaussian field distribution, which has the optimal coupling to the PCF mode. This is done through an iterative process, where we optimise the alignment, the polarisation, the phase, and the width of the gaussian field. We then examine the behaviour of the coupling coefficient and the width of the gaussian field, as a function of normalized frequency, $\Lambda / \lambda$, where $\lambda$ is the free space wavelength of the light, and normalized hole size, d. Fig. 3 shows the normalized spotsize, $\omega / \Lambda$, for the fundamental mode of PCFs as a function of the normalized frequency. Notice that there is a difference between standard fiber terminology and PCF terminology. The normalized frequency is, when used on PCFs and in this article, $\Lambda / \lambda$, which is proportional to the frequency of the light. The spotsize is defined as the 1/e-radius of the electromagnetic field, i.e., the $1 / \mathrm{e}^{2}$-width of the power. For the larger hole sizes, the fibers are no longer endlessly single moded, this is indicated using dashed lines for the multi-mode range.

Fig. 3 shows the spotsize of gaussian beam optimized with respect to coupling to PCFs. The overlap between fundamental PCF mode and optimized gaussian beams is more than $95 \%$ for PCF having $d / \Lambda>0.4$ for $\Lambda / \lambda>1$ (not shown in figure). The results illustrate that the spotsize is a decreasing function of both the hole size and the normalized frequency. The reason for this hole size dependency is that for larger holes, for a given frequency, more power is present in the air holes, creating a larger index contrast between the core and the cladding. Furthermore, the physical size of the core region decreases as the boundaries of the neighbouring holes surrounding the core moves towards the centre of the fiber. The frequency dependence is caused by the same mechanism observed in standard fibers; the shorter wavelength results in a mode, which is closer and closer confined to the core of the fiber, but has a increasingly smaller index contrast. As a further point, the PCFs are found to become multi

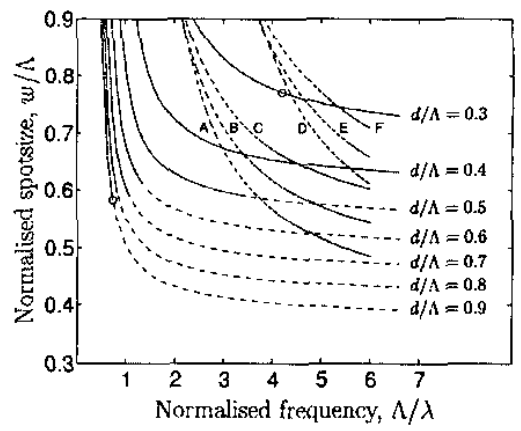

ThGG11 Fig. 3. Normalized spotsize $w / \Lambda$ as a function of normalized frequency, $\Lambda / \lambda$. The curve parameter is the normalized hole size $d / \Lambda$. Regions where the fibers are multimode are indicated by dashed curves. The figure illustrates further the normalized spotsize $w / \Lambda$ for two sets of step-index fibers (SIF) with different refractive index step: A,B,C having $\Delta \mathrm{n}=0.005$, and $\mathrm{D}, \mathrm{E}, \mathrm{F}$ having $\Delta \mathrm{n}=0.015$. Core sizes are $A, D: 0.6 \Lambda, B, E$ : $0.7 \Lambda, D, F: 0.8 \Lambda$ 

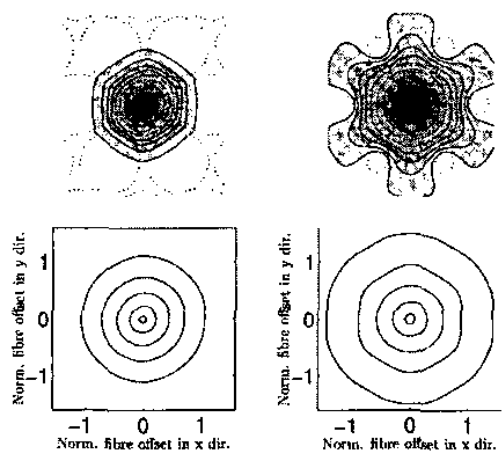

ThGG11 Fig. 4. Top figures: Mode field distributions of PCFs with $\mathrm{d} / \Lambda=0.9$ at $\Lambda / \lambda=0.74$ (left) and $d / L=0.3$ at $\Lambda / \lambda=4.18$ (right). Bottom figures: Directional variation in the offset for coupling between PCFs and optimum SIFs.

moded for spotsizes below approximately $0.6 \Lambda$. Fig. 3 further shows the spotsize and optimum design parameters for two sets of SIFs having refractive index step of 0.005 and 0.0015 , respectively. As an important result, the figure shows that optimum coupling between PCFs and SIFs with realistic refractive index contrasts, may only be obtained for PCFs with relatively low $d / \Lambda$ (d $/ \Lambda$ below 0.5 ). Additionally, for optimum coupling, the PCFs should be designed in the regime of $\Lambda / \lambda$ $>3$.

Finally, Fig. 4 illustrates directional offset variation for coupling losses between PCFs and optimum SIFs. Contour levels are in relation to the maximum coupling coefficient and are at levels: $0.99,0.95,0.9,1 / \mathrm{e}, 1 / \mathrm{e} 2$. This result indicates that the angular dependency of transversal offset when coupling to PCFs is of limited importance, despite the hexagonal distribution of the PCF mode fields.

\section{Summary}

In this work we have analyzed the correspondence between the fundamental mode of PCFs and gaussian modes as a function of frequency, pitch, and air hole size. Such analysis provides insight into design space regions of PCFs, where low-loss coupling to standard fibers may be obtained.

\section{References}

1. Broeng et al., Pure and Applied Optics, 1, 477-482 (1999).

2. Bennet et al., ECOC '99, pages 120-123, Sept., (1999).

3. Lizier et al. IEEE Photonics Technology Letters, 13 (8), p. 794-796 (2001).
Combined multimode and singlemode fiber

Bengt Lindström, Focotech Svenska $A B$, Kontoristvägen 6, SE-12357 Farsta, Sweden

Bernt Sundström, Mats Granberg, Leif Stensland and Torsten Augustsson, Ericsson

Microelectronics AB, SE-164 81 Kista, Sweden,

Email: bernt.sundstrom@mic.ericsson.se

Anders Djupsjöbacka, Ericsson Telecom AB, SE12625 Stockholm, Sweden

Anders Larsson, Bertil Arvidsson and Thomas $\mathrm{K}$. Ericsson, Ericsson Network Technology AB, Kabelvägen 1, SE-824 82 Hudiksvall, Sweden

\section{Introduction}

Multimode (MM) or singlemode (SM) fibers for short haul have been discussed for decades. ${ }^{1-3}$ Cables with both fiber types have been installed, with the possibility to start using multimode and then upgrade to singlemode fiber when needed. In this paper we propose, for the first time to our knowledge, a combined multimode and singlemode fiber (MM-SM-fiber) for telecommunication and FTTH applications (Swedish patent application filed 2001-07-12, No. 0102509-7). We have superposed a singlemode core concentrically on a multimode core index profile, obtaining a fiber with the advantages of both fibers. When the fiber is connected to multimode fibers at both ends, it works as a multimode fiber. When it is connected to singlemode fibers at both ends, this fiber has the same high bandwidth as a standard singlemode fiber. Upgrading or downgrading is very simple and cheap, as the only things to be changed are the connecting fibers. Furthermore, this fiber can be used for duplex communication, e.g., the SM-core downstream and the MM-core upstream.

\section{Manufacturing}

A preform was manufactured by the MCVD method. A sufficient number of multimode core layers were first deposited, followed by the singlemode core layers. Special care was taken to prevent inhomogeneities during the deposition. The collapse process is delicious to prevent too much diffusion and avoid cracking of the preform. Two fibers, each $2 \mathrm{~km}$ long, were drawn in a normal way. The obtained index profile is shown in Fig. 1.

\section{Measurements}

The following measurements were performed to test the MM and SM properties: MM-OTDR, MM-bandwidth, SM-OTDR, SM-cutback, SM chromatic dispersion, $10 \mathrm{Gbit} / \mathrm{s}$ eye-diagram and $10 \mathrm{Gbit} / \mathrm{s}$ BER-measurement. When MM meas-

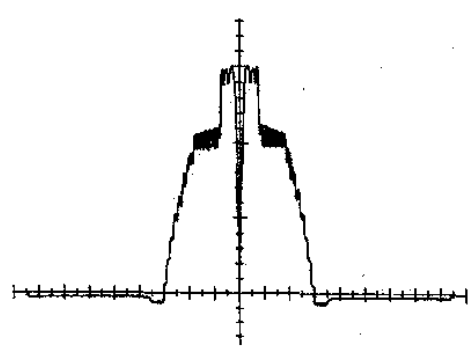

ThGG12 Fig. 1. Index profile of the MMSM-preform. urements were performed, MM-fibers were spliced or connected to the MM-SM-fiber, and for SM measurements, SM-fibers were spliced to the MM-SM-fiber

A MM-OTDR measured a MM attenuation of $2.7 \mathrm{~dB} / \mathrm{km}$ at $905 \mathrm{~nm}$ wavelength. MM-bandwidth was measured using a Tektronix OF192 bandwidth test set. For a $2 \mathrm{~km}$ length of fiber, a MM-bandwidth around $100 \mathrm{MHz}$ was measured at $1300 \mathrm{~nm}$, using a Gaussian curve-fit, corresponding to about $200 \mathrm{MHz} \cdot \mathrm{km}$. Without the Gaussian curve-fit, the instrument showed around $12 \mathrm{MHz}$. The reason is not known, but the $\mathrm{MM}$ index profile was not optimized so far. However, high MM-bandwidth is not necessary as upgrading to SM-operation is an easy task.

The SM attenuation was measured using SMOTDR and SM-cutback. The SM-OTDR straight line showed a uniform attenuation with a relatively high loss, $1.17 \mathrm{~dB} / \mathrm{km}$ at $1300 \mathrm{~nm}$ and 0.72 $\mathrm{dB} / \mathrm{km}$ at $1550 \mathrm{~nm}$. The cause for this is unknown. The SM-cutback measurement was obscured at $1300 \mathrm{~nm}$ by a high cutoff wavelength about $1380 \mathrm{~nm}$, and the absolute value is also uncertain as one cannot attach a SM-fiber to the short length output end without introducing unknown losses. SM chromatic dispersion was measured in a standard SM measurement instrument from EG\&G and showed a small shift to $\lambda_{0}=1390$ nm, but was otherwise normal, see Fig. 2 .

In the eye measurements an OC-192/STM-64, i.e., $9.95328 \mathrm{Gbit} / \mathrm{s}$, NRZ-modulated optical signal with a PRBS-31 pattern was used. The first eye diagram was taken with an optical oscilloscope directly connected to the transmitter at a mean input power of $-6 \mathrm{dBm}$, Fig. 3a. The second eye was taken with the MM-SM-fiber between the transmitter and the optical oscilloscope, Fig. 3b. With the MM-SM-fiber installed, the Q-value degraded from 11.5 to 10.5 (linear). The latter $(2-$ value corresponds to a bit error-rate (BER) of $5 \cdot 10^{-26}$.

In the bit error-rate measurement, the same signal as in the eye-diagrams measurement was used. Here, the optical signal was fed into an $\mathrm{OC}$ 192/STM-64 receiver that was connected to a BER analyzer. The receiver used was a PIN/GaAsPHEMT hybrid with no pre-amplification, thereby the $1 \cdot 10^{-9}$-sensitivity of $-20.5 \mathrm{dBm}$. In Fig. 4, the BER-curves for the back-to-back measurement and the MM-SM-fiber measurement is plotted together with a reference curve representing a thermal noise curve. From the BER-curves it can be seen that the power penalty for the MMSM-fiber is about $0.5 \mathrm{~dB}$ compared to the backto-back case.

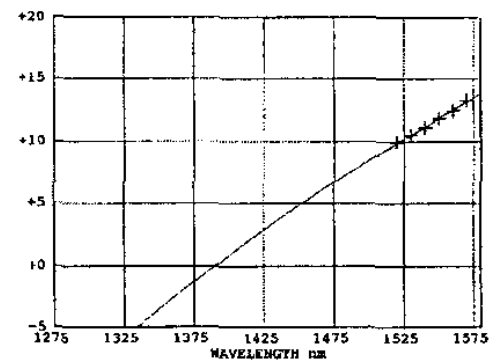

ThGG12 Fig. 2. Singlemode chromatic dispersion in $\mathrm{ps} / \mathrm{nm} \cdot \mathrm{km}$. 